\title{
Dispersive waves in functionally graded plates
}

\author{
Alla Ilyashenko $^{1}$ and Sergey Kuznetsov ${ }^{1,2}$ \\ ${ }^{1}$ Moscow State University of Civil Engineering, Yaroslavskoe shosse, 26, Moscow, 129337, Russia \\ ${ }^{2}$ Institute for Problems in Mechanics, Moscow, 119526, Russia
}

\begin{abstract}
The dispersion waves propagating in anisotropic functionally graded (FG) plates with arbitrary transverse heterogeneity and arbitrary elastic monoclinic anisotropy are analysed within a recently developed sixdimensional formalism. The dispersion relation is obtained for all modes of dispersive harmonic waves propagating in an unbounded plate.
\end{abstract}

\section{Introduction}

Functionally graded (FG) materials with transverse inhomogeneity can considerably change material acoustic properties. That may result in filtering specific types of acoustic signals at some frequencies $[1-13]$. These properties of wave propagation in FG plates are of particular interest in various NDT applications [14 - 16]. However, until now there were no closed form analytical solutions for dispersion equations suitable for Lamb waves propagating in FG plates with arbitrary elastic (monoclinic) anisotropy and arbitrary transverse inhomogeneity.

The developed methodology relies on a previously developed six-dimensional formalism, known as Cauchy formalism [17 - 21] for deriving the dispersion equations for stratified plates or stratified halfplanes with homogeneous anisotropic layers of arbitrary elastic monoclinic anisotropy and the transverse inhomogeneity.

The modification for the FG transverse inhomogeneity utilizes a specially constructed matrix exponential solution to the matrix six-dimensional ODE with the non-constant coefficients. It is assumed that within the particular layer the inhomogeneity is continuously differentiable with respect to the transverse variable.

\section{Basic equations}

Herein, the main equations for constructing the solution for Lamb waves in a functionally graded plate are derived.

\subsection{Equations of motion}

The linear equations of motion for anisotropic inhomogeneous material can be written in a form

$$
\operatorname{div} \mathbf{C}(\mathbf{x}) \cdot \nabla \mathbf{u}(\mathbf{x}, t)=\rho(\mathbf{x}) \dot{\mathbf{u}}(\mathbf{x}, t),
$$

where $\mathbf{C}(\mathbf{x})$ is the fourth-order elasticity tensor assumed to be strongly elliptic 


$$
\underset{\left.\mathbf{x} \in\lrcorner^{3} \mathbf{m}, \mathbf{n} \in\right\lrcorner^{3}, \mathbf{m}, \mathbf{n} \neq 0}{\forall \mathbf{x}} \quad \forall \mathbf{m}, \mathbf{n} \quad \mathbf{m} \otimes \mathbf{n} \cdot \cdot \mathbf{C}(\mathbf{x}) \cdot \cdot \mathbf{n} \otimes \mathbf{m}>0
$$

and $\left.\mathbf{C}(\mathbf{x}) \in C^{1}(\urcorner^{3}\right)$, i.e. $\mathbf{C}(\mathbf{x})$ is continuously differentiable in $\rfloor^{3}$.

Hereafter, propagation of harmonic Lamb wave in a plate with transverse inhomogeneity specified by transverse dimensionless complex coordinate

$$
x=\operatorname{ir} \mathbf{x} \cdot \mathbf{v},
$$

where $v$ is the unit normal to the median plane; $r$ is the wave number having dimension $l^{-1}$ and $i=\sqrt{-1}$; see Fig. 1. Origin of the global coordinate system belongs to the median plane $\Pi_{v}$.

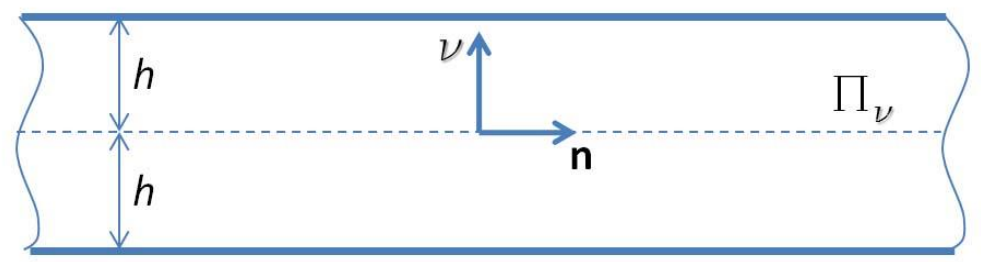

Fig. 1. Plate of thickness $2 h$; unit vectors $\mathbf{n}$ and $\boldsymbol{v}$ indicate direction of propagation and normal to the median plane $\Pi_{v}$ respectively

The following wave representation for Lamb wave is adopted

$$
\mathbf{u}(\mathbf{x}, t)=\mathbf{m}(x) e^{i r(\mathbf{n} \cdot \mathbf{x}-c t)},
$$

where $\mathbf{m}$ is (unknown) variation of the vector wave amplitude across thickness of the plate; $\mathbf{n}$ is the unit vector indicating direction of propagation; $c$ is the independent of $x$ phase velocity of Lamb wave; $t$ is time.

Substituting representation (1.4) into equations of motion, yields

$$
\left(\mathbf{A}_{1}(x) \frac{d^{2}}{d x^{2}}+\mathbf{A}_{2}(x) \frac{d}{d x}+\mathbf{A}_{3}(x)\right) \cdot \mathbf{m}(x)=0
$$

where

$$
\begin{aligned}
& \mathbf{A}_{1}(x)=\mathbf{v} \cdot \mathbf{C}(x) \cdot \mathbf{v} \\
& \mathbf{A}_{2}(x)=\mathbf{v} \cdot\left(\frac{d}{d x} \mathbf{C}(x)\right) \cdot \mathbf{v}+\mathbf{v} \cdot \mathbf{C}(x) \cdot \mathbf{n}+\mathbf{n} \cdot \mathbf{C}(x) \cdot \mathbf{v} \\
& \mathbf{A}_{3}(x)=\mathbf{n} \cdot \mathbf{C}(x) \cdot \mathbf{n}-\rho(x) c^{2} \mathbf{I}
\end{aligned}
$$

In (1.6) I denotes unit diagonal matrix. Note, that strongly ellipticity condition (1.2) ensures

$$
\operatorname{det} \mathbf{A}_{1}(x)>0
$$

and

$$
\operatorname{det} \mathbf{A}_{3}(x)>0
$$

provided phase velocity $c$ satisfies the following condition

$$
c<\sqrt{\frac{\lambda_{3}(\mathbf{n} \cdot \mathbf{C}(x) \cdot \mathbf{n})}{\rho(x)}},
$$

where $\lambda_{3}$ is the smallest eigenvalue of $\mathbf{n} \cdot \mathbf{C}(x) \cdot \mathbf{n}$. Hereafter, it is assumed that the condition (1.9) is satisfied. 


\subsection{Formalism Cauchy}

Introducing a new variable

$$
\mathbf{w}(x)=\frac{d}{d x} \mathbf{m}(x)
$$

yields equations of motion in terms of two unknown vector functions $\mathbf{m}$ and $\mathbf{w}$ :

$$
\begin{aligned}
& \frac{d}{d x} \mathbf{m}(x)=\mathbf{w}(x) \\
& \frac{d}{d x} \mathbf{w}(x)=-\mathbf{A}_{1}^{-1}(x) \cdot \mathbf{A}_{3}(x) \cdot \mathbf{m}(x)-\mathbf{A}_{1}^{-1}(x) \cdot \mathbf{A}_{2}(x) \cdot \mathbf{w}(x)
\end{aligned}
$$

where it is assumed that acoustical tensor $\mathbf{A}_{1}(x)$ is invertible.

Introducing a new 6-dimensional vector

$$
\mathbf{Y}(x)=\left(\begin{array}{l}
\mathbf{m}(x) \\
\mathbf{w}(x)
\end{array}\right)
$$

allows us to rewrite Eqs. (1.11) in the following form

$$
\frac{d}{d x} \mathbf{Y}(x)=\mathbf{G}(x) \cdot \mathbf{Y}(x),
$$

where $\mathbf{G}(x)$ is a six-dimensional matrix:

$$
\mathbf{G}(x)=\left(\begin{array}{cc}
\mathbf{0} & \mathbf{I} \\
-\mathbf{A}_{1}^{-1}(x) \cdot \mathbf{A}_{3}(x) & -\mathbf{A}_{1}^{-1}(x) \cdot \mathbf{A}_{2}(x)
\end{array}\right)
$$

Equation (1.13) is the main equation of Cauchy formalism, matrix $\mathbf{G}$ will be called as the fundamental matrix. Conditions (1.2) and (1.9) ensure

$$
\operatorname{det} \mathbf{G}(x)=\operatorname{det} \mathbf{A}_{1}^{-1}(x) \operatorname{det} \mathbf{A}_{3}(x)>0
$$

\subsection{Boundary conditions}

Traction-free boundary conditions have the form

$$
\left.\mathbf{t}_{v}(\mathbf{x}, t) \equiv \mathbf{v} \cdot \mathbf{C}(x) \cdot \nabla \mathbf{u}(\mathbf{x}, t)\right|_{x= \pm i r h}=0,
$$

where the plate thickness is $2 h$. Substituting representation (1.4) into boundary conditions (1.16) with account of notations (1.6), (1.10) yields

$$
\mathbf{A}_{1}(x) \cdot \mathbf{w}(x)+\left.\mathbf{A}_{4}(x) \cdot \mathbf{m}(x)\right|_{x= \pm i r h}=0
$$

where

$$
\mathbf{A}_{4}(x)=\mathbf{v} \cdot \mathbf{C}(x) \cdot \mathbf{n}
$$

Lastly, conditions (1.17) can be rewritten in terms of vector $\mathbf{Y}(x)$ :

$$
\left.\mathbf{t}_{v}(x) \equiv\left(\mathbf{A}_{4}(x), \mathbf{A}_{1}(x)\right) \cdot \mathbf{Y}(x)\right|_{x= \pm i r h}=0
$$

\subsection{Matrix equation}

Let $\mathbf{E}$ be a matrix satisfying the same Eq. (1.13):

$$
\frac{d}{d x} \mathbf{E}(x)=\mathbf{G}(x) \cdot \mathbf{E}(x)
$$

Matrix $\mathbf{E}$ is assumed to be non-degenerate.

If such a matrix exists, then any vectorial solution $\mathbf{Y}(x)$ from Eq. (1.13) due to linearity of the problem takes the form 


$$
\mathbf{Y}(x)=\mathbf{E}(x) \cdot \vec{C},
$$

where $\vec{C}$ is a 6-dimensional vector of unknown coefficients, defined by boundary conditions (1.16).

Equation (1.20) can obviously be rewritten in equivalent form

$$
\left(\frac{d}{d x} \mathbf{E}(x)\right) \cdot \mathbf{E}^{-1}(x)=\mathbf{G}(x)
$$

\subsection{Matrix solution}

Applying matrix function analysis [14] allows constructing matrix solution for Eq. (1.22) in the form:

$$
\mathbf{E}(x)=e^{\mathbf{F}(x)+\mathbf{A}},
$$

where

$$
\mathbf{F}(x) \equiv \int \mathbf{G}(x) d x
$$

stands for (any) antiderivative of $\mathbf{G}(x)$. It will be shown later, that an arbitrary (constant) matrix appearing in (1.23) does not influence the final result.

Remark 2.1. Direct verification reveals that matrix (1.23) satisfies Eq. (1.22). The righthand side of expression (1.23) shows that matrix $\mathbf{E}(x)$ is non-degenerate at the phase velocity satisfying condition (1.9), that is due to (1.15). Now, combining (1.21) and (1.23) the vector-valued general solution $\mathbf{Y}$ can be constructed.

\section{Basic equations}

Substituting the general solution at $x=+i r h$ into representation (1.21) with account of (1.12), yields

$$
\left.\cdot \mathbf{E}(x) \cdot \vec{C}\right|_{x=+i r h}=\mathbf{Y}(i r h),
$$

from where

$$
\vec{C}=\mathbf{E}^{-1}(i r h) \cdot \mathbf{Y}(i r h)
$$

Eq. (2.1) yields

$$
\mathbf{Y}(-i r h)=\mathbf{E}(-i r h) \cdot \underbrace{\mathbf{E}^{-1}(i r h) \cdot \mathbf{Y}(i r h)}_{\vec{C}}
$$

The following $6 \times 6$ matrix is needed for the subsequent analysis

$$
\mathbf{Z}(x)=\left(\begin{array}{cc}
\mathbf{I} & 0 \\
\mathbf{A}_{4}(x) & \mathbf{A}_{1}(x)
\end{array}\right)
$$

Note, that matrix $\mathbf{Z}(x)$ is invertible at any $x$, since $\operatorname{det} \mathbf{Z}=\operatorname{det} \mathbf{A}_{1}>0$ due to strong ellipticity condition (1.2). Thus, matrix $\mathbf{Z}(x)$ can be considered as one-to-one mapping in $\mathrm{R}^{6}$, and taking into account (1.12), (1.19) 


$$
\left(\begin{array}{l}
\mathbf{m}(x) \\
\mathbf{t}_{v}(x)
\end{array}\right)=\mathbf{Z}(x) \cdot\left(\begin{array}{l}
\mathbf{m}(x) \\
\mathbf{w}(x)
\end{array}\right)
$$

Now, both surface displacement magnitude $\mathbf{m}$ and surface traction $\mathbf{t}_{v}$ at $x=-i r h$, can be written in terms of matrix $\mathbf{Z}$ and expressions (2.3) and (2.5)

$$
\left(\begin{array}{c}
\mathbf{m}(-i r h) \\
\mathbf{t}_{v}(-i r h)
\end{array}\right)=\mathbf{T}(i r h) \cdot\left(\begin{array}{l}
\mathbf{m}(i r h) \\
\mathbf{t}_{v}(i r h)
\end{array}\right) \text {, }
$$

where

$$
\mathbf{T}(i r h) \equiv \mathbf{Z}(-i r h) \cdot \mathbf{E}(-i r h) \cdot \mathbf{E}^{-1}(i r h) \cdot \mathbf{Z}^{-1}(i r h)
$$

Useful for computation purposes formula flows out from analyzing composition $\mathbf{E}(-i r h) \cdot \mathbf{E}^{-1}(i r h)$, revealing

$$
\mathbf{E}(-i r h) \cdot \mathbf{E}^{-1}(i r h)=e^{\mathbf{F}(-i r h)-\mathbf{F}(i r h)}=\exp \left(-\int_{-i r h}^{i r h} \mathbf{G}(x) d x\right)
$$

Thus, the composition $\mathbf{E}(-i r h) \cdot \mathbf{E}^{-1}($ irh $)$ does not contain an arbitrary (constant) matrix A ; see Eq. (1.23).

Remark 3.1. Since surface traction fields in (2.6) vanish at $x= \pm i r h$, the following matrix operator acting in $\mathrm{R}^{3}$ space

$$
R^{3} \stackrel{(\mathbf{0}, \mathbf{I}) \cdot \mathbf{T} \cdot\left(\begin{array}{l}
\mathbf{I} \\
\mathbf{0}
\end{array}\right)}{\longrightarrow} R^{3}
$$

from 3D space of surface displacements and vanishing surface-tractions on the "upper" surface to 3D space of surface-tractions on the "bottom" surface, should be degenerate to ensure existence of a non-trivial displacement magnitude on the "upper" surface resulting in vanishing surface tractions on the "bottom" surface. The latter is equivalent

$$
\operatorname{det}\left((\mathbf{0}, \mathbf{I}) \cdot \mathbf{T} \cdot\left(\begin{array}{l}
\mathbf{I} \\
\mathbf{0}
\end{array}\right)\right)=0
$$

Equation (2.10) is the desired dispersion equation for a plate with free boundaries. The dispersion equation (2.10) coincides with one obtained for a homogeneous anisotropic plate [13].

\section{Conclusions}

Propagation of harmonic Lamb waves in plates made of functionally graded materials (FGM) with transverse inhomogeneity is analyzed by applying and modifying Cauchy sixdimensional formalism previously developed for studying Lamb wave propagation in homogeneous or stratified anisotropic plates with arbitrary elastic anisotropy [13, 14]. For FG plates with arbitrary transverse inhomogeneity closed form implicit solution to dispersion equation is derived and analyzed.

Closed form implicit solutions for anisotropic FG plates with exponential inhomogeneity are constructed and compared with the corresponding implicit solutions for homogeneous plates, revealing their resemblance. 
Authors thank the Russian Science Foundation for Basic Research (Grants 17-08-00311 and 18-5841001) and The Presidium of Russian Academy of Sciences (Programs Nanomaterials and Supercomputers) for partial financial support.

\section{References}

1. M. B. Amor, M. H. B. Ghozlen, Ultrasonics 4905, 1 (2014)

2. X. Han, G. R. Liu, K. Y. Lam, T. Ohyoshi, J. Sound Vibr. 236, 307 (2000)

3. V. Vlasie, M. Rousseau, NDT\&E Int. 37, 633 (2004)

4. C. Baron, S. Naili, J. Acoust. Soc. Am., 127, 1307 (2010)

5. A. L. Shuvalov, E. Le Clezio, G. Feuillard, Int. J. Eng. Sci., 46, 929 (2008)

6. A. L. Shuvalov, O. Poncelet and M. Deschamps, Wave Motion 40, 413 (2004)

7. A. L. Shuvalov and A. G. Every, Wave Motion 36, 257 (2002)

8. L. Wang, F. G. Yuan, Composites Sci. Technol. 67, 1370 (2007)

9. S.-E. Hebaz, F. Benmeddour, E. Moulin, J. Assaad, J. Acoust. Soc. Am. 143, 460 (2018)

10. X. Han, G.R. Liu, Z.C. Xi, \& K.Y. Lam, Int. J. Num. Meth. Eng. 53, 653 (2002)

11. Chao Xu, Zexing Yu, Adv. Mech. Eng. 9, 1 (2017)

12. N. Nanda, S. Kapuria, Composite Struct. 132, 310 (2015)

13. J. E. Lefebvre et al., IEEE T Ultrason. Ferr., 48, 1332 (2001)

14. R. V. Craster, J. D. Kaplunov, and A. V. Pichugin, Proc. R. Soc. A 466, 2341 (2010)

15. R. V. Craster, L. M. Joseph, J. D. Kaplunov, Wave Motion 51, 581 (2014)

16. Chao Xu, Zexing Yu, Adv. Mech. Eng. 9, 1 (2017)

17. S. V. Kuznetsov, Acoust. Physics 60, 95 (2014)

18. S. V. Kuznetsov, Quart. Appl. Math. 62, 749 (2004)

19. S. V. Kuznetsov, Quart. Appl. Math. 64, 153 (2006)

20. S. V. Kuznetsov, Acoustical Physics, 56, 877 (2010)

21. R. V. Goldstein, A. V. Ilyashenko, S. V. Kuznetsov, Math. Models Comp. Simul. 10, 308 (2018) 